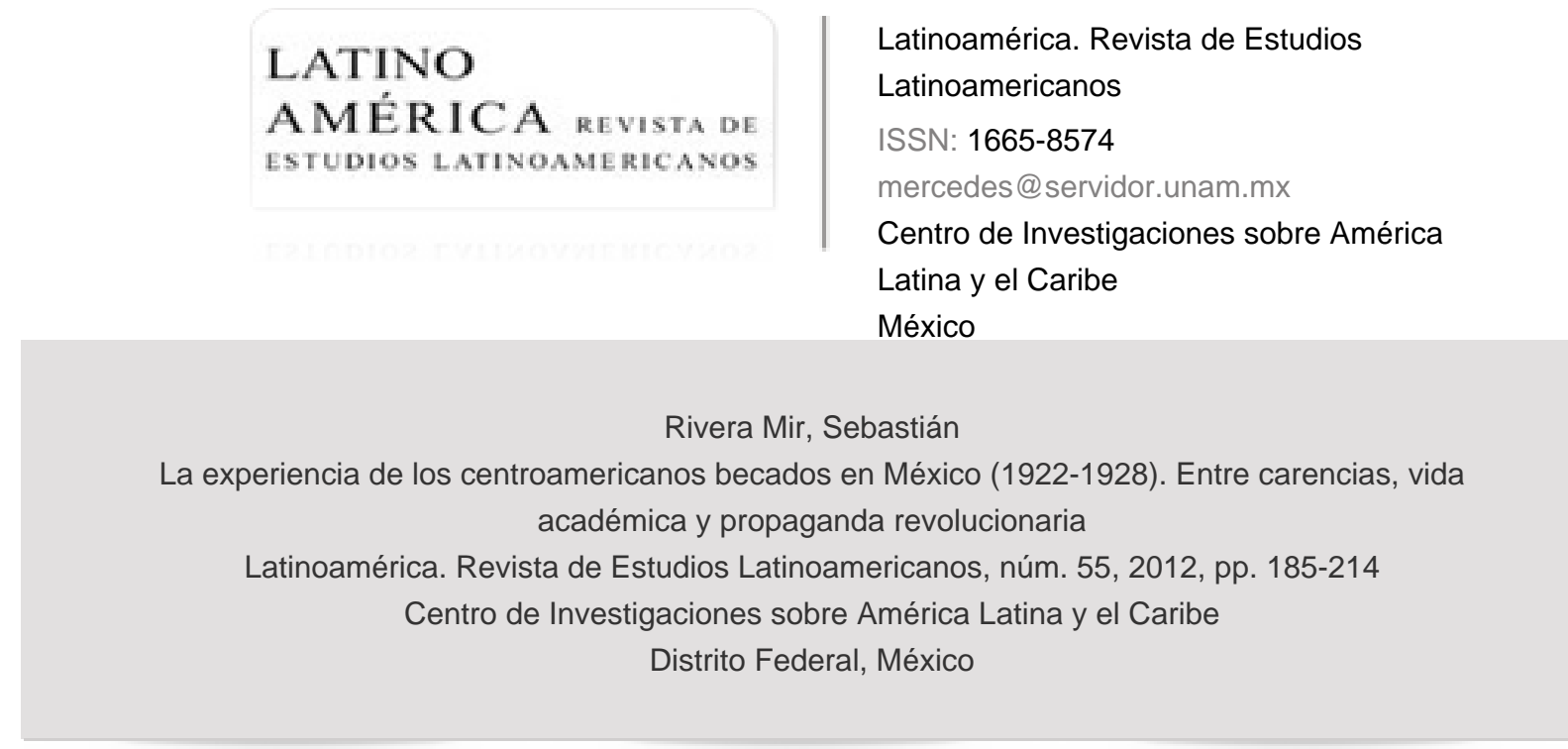

Disponible en: http://www.redalyc.org/articulo.oa?id=64024698008

Cómo citar el artículo

- Número completo

- Más información del artículo

- Página de la revista en redalyc.org

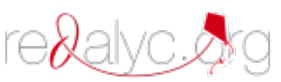

Sistema de Información Científica

Red de Revistas Científicas de América Latina, el Caribe, España y Portugal Proyecto académico sin fines de lucro, desarrollado bajo la iniciativa de acceso abierto 


\title{
La experiencia de los centroamericanos becados en México (1922-1928). Entre carencias, vida académica y propaganda revolucionaria
}

\author{
Sebastián Rivera Mir*
}

Resumen: Esta investigación analiza el arribo a México de un grupo de becarios centroamericanos durante los gobiernos de Alvaro Obregón y Plutarco Elías Calles. Estos apoyos buscaron consolidar los lazos culturales de la región y exponer una imagen positiva de México. Este trabajo intenta conjugar estas políticas estatales con las vivencias de los becarios, sus carencias, sus esfuerzos académicos y sus decepciones. Se trata de observar cómo los planes gubernamentales latinoamericanistas entraron en diálogo con las metas de los estudiantes y cómo dicho proceso significó un avance en la consolidación del sistema mexicano de intercambio académico.

Palabras clave: Becarios-centroamericanos, Políitcas culturales, Diplomacia, Intercambio académico.

ABSTRACT: This research analyzes the arrival to Mexico of a Central-Americans fellows group during the governments of Alvaro Obregón and Plutarco Elías Calles. These grants attempted to consolidate the cultural ties of the region and expose a positive image of Mexico. This work tries to combine these state policies, with the experiences of students, their mishaps, their academic endeavors and their disappointments. The principal aim is to observe how the plans of the government of Mexico, based on latinoamerican union, holded talks with the students and their goals and how this process was a step forward to consolidate the academic exchange mexican system.

Key words: Fellows-Central-Americans, Cultural Policy, Diplomacy, Academic Exchange.

"El Colegio de México (srivera@colmex.mx). 


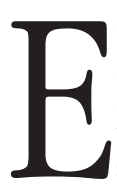

110 de octubre de 1928, Plutarco Elías Calles envió un memorándum al entonces subsecretario de Educación Pública, Moisés Sáenz, en el que le pedía: "He de merecer a usted se sirva librar sus apreciables órdenes a fin de que los jóvenes Horacio Canales y Reynaldo Lacayo queden favorecidos con las becas que el Congreso de la Unión acordó dar a los estudiantes centroamericanos". ${ }^{1}$

En primer lugar, con estas palabras el presidente de México, en las postrimerías de su mandato oficial, intentaba permitir que los jóvenes ingresaran a la Universidad Nacional, petición en nada lejana a sus intereses pedagógicos. El Jefe Máximo había sido profesor antes de embarcarse en las agitadas aguas de la revolución. Como segundo objetivo, podemos observar que detrás de esta misiva se encuentra la búsqueda de mejorar un aparato educativo que muchas veces dependió demasiado de las "apreciables órdenes" de las autoridades. La vinculación efectiva entre las distintas esferas del poder político era algo necesario para que el Estado posrevolucionario se consolidara. Y por último, en la carta de Calles también vemos que existe una clara direccionalidad de estas becas: son para estudiantes centroamericanos. La política cultural del presidente mexicano miraba hacia los vecinos del sur.

El objetivo general de este trabajo es observar cómo se desarrolló el proceso de entrega de becas a estudiantes centroamericanos durante los primeros años del Estado posrevolucionario. ${ }^{2}$ A mi modo de ver, las pensiones ${ }^{3}$ tuvieron diversos usos durante este periodo, pero en la mayoría de los casos permitieron entrelazar una política de propaganda cultural revolucionaria, tanto en el interior

1 Memorándum de Plutarco Elías Calles a Moisés Sáenz, 10 de octubre, 1928, Fideicomiso Archivos Plutarco Elías Calles-Fernando Torreblanca (en adelante FAPECFT), Fondo Plutarco Elías Calles, exp. 13, inv. 5213, leg. 1.

2 Me interesa ver especialmente el caso de Latinoamérica, aunque considero importante establecer vínculos con el proceso en su conjunto, ya que en muchos casos se estaba mirando hacia todos lados, Europa, Estados Unidos, incluso Japón, para establecer una nueva institucionalidad. También es relevante destacar que en la relación entre México y América Latina, el intercambio universitario involucró mecanismos de financiamiento, por lo que becas e intercambio fueron prácticamente sinónimos. En sus relaciones con otras regiones del mundo, esta situación no fue siempre así.

3 Para el periodo ambos conceptos pueden considerarse sinónimos. 
como en el exterior del país, con la consolidación de instituciones estatales destinadas a estos fines.

Entre los diversos usos de la "cultura" como mecanismo propagandístico, muy pocos trabajos historiográficos se han detenido a reflexionar sobre el sistema de becas. Algunos autores hacen énfasis en los viajes de intelectuales, otros en el rol de las publicaciones y las escuelas de verano, o incluso en los murales como forma de plasmar las intenciones de la Revolución en el extranjero. ${ }^{4}$ Sin embargo, los inicios de un balbuceante sistema de intercambio académico han sido considerados de menor relevancia, especialmente si estamos hablando de las relaciones entre México y América Latina.

Los marcos temporales de mi trabajo son necesariamente difusos. Podríamos pensar en los límites desarrollados por la historiografía: las primeras becas del gobierno posrevolucionario, según Claude Fell, fueron entregadas por Álvaro Obregón en 1922, ${ }^{5}$ mientras que María Cecilia Zuleta menciona que ya hacia 1936 el sistema de intercambio entró en una etapa de consolidación, especialmente, aunque no de manera exclusiva, debido a la llegada de los exiliados españoles. ${ }^{6}$ Sin embargo, si pensamos estas calendarizaciones desde la posición de los becarios, las fechas no son tan claras, no sólo porque muchos permanecieron estudiando un tiempo irregular, sino que además algunos proyectaron su vida en México más allá de las estructuras formales del sistema educativo. De ese modo, esta investigación exige conjugar varias temporalidades, los periodos de gobierno, los procesos educativos universitarios, la gestación de la instrumentalidad institucional detrás del sistema de pensiones y, también, el ritmo de vida de los propios becarios. ${ }^{7}$ En esta perspectiva, me parece que hacia fines de la dé-

4 Véanse Pablo Yankelevich, "En la retaguardia de la Revolución mexicana. Propaganda y propagandistas mexicanos en América Latina, 1914-1920", en Mexican Studies/Estudios Mexicanos, vol. 15, núm.1, The University of California Press, invierno de 1999, pp. 35-71; Alicia Azuela de la Cueva, Arte y poder. Renacimiento artístico y revolución social. México 1910-1945, México, El Colegio de Michoacán/FCE, 2005. Especialmente el capítulo "La diplomacia por otros medios".

5 Claude Fell, José Vasconcelos. Los años del águila, México, unam, 2009, p. 351.

6 María Cecilia Zuleta, Los extremos de Hispanoamérica. Relaciones, conflictos y armonías entre México y el Cono Sur, 1821-1990, México, SRE, 2008.

7 Véase Engracia Loyo, Gobiernos revolucionarios y educación popular en México, 1911-1928, México, El Colegio de México, 1999. 
cada de 1920 hay un punto de inflexión donde coinciden procesos como el cambio en la política internacional de México, la modificación del estatus de la Universidad Nacional, la graduación de muchos de los primeros becados, entre otros elementos. ${ }^{8}$ Por lo tanto, si bien enmarcaré este trabajo entre 1922 y 1928 , de todas maneras pretendo mantener una flexibilidad temporal que permita potenciar el análisis.

Como podemos percibir en la carta de Plutarco Elías Calles, las fuentes de información en este ámbito son amplias, pero a la vez también muy fragmentadas. El intercambio académico era un tema que no sólo preocupaba al presidente en plena guerra cristera; la Secretaría de Educación Pública (SEP) manejaba algunas aristas, mientras la Secretaría de Relaciones Exteriores (SRE) se encargaba de otros aspectos. Por su parte, la Universidad, pese a construir una buena parte de los planes de intercambio académico, dependió en aquel momento de quién otorgaba las pensiones. Incluso, estas relaciones involucraron a estudiantes militares, lo que motivó la participación de entidades estatales muchas veces no mencionadas como impulsoras del ámbito estudiantil, como el Colegio Militar.

Si bien la perspectiva tomada en este trabajo se refiere fundamentalmente a la recepción de estudiantes extranjeros en México, debemos considerar que estos procesos fueron paralelos al envío de pensionados mexicanos al exterior. Mientras en estas tierras se exigía a los centroamericanos enviar sus informes de manera rigurosa a la SEP, en octubre de 1923, se definían como indispensables los reportes de conducta de los alumnos pensionados en el extranjero, para revalidar sus becas. De hecho, un acuerdo presidencial establecía que "[...] los respectivos ministros o cónsules estarán encargados de vigilar la conducta y aprovechamiento de los pensionados y rendir los informes necesarios para que lleguen a conocimiento de la Secretaría de Educación Pública".

8 Renate Marsiske, "Los estudiantes en la Universidad Nacional de México: 1910-1928", en Varios autores, Los estudiantes. Trabajos de historia y sociología, México, CESU-unam, 1989.

9 Oficio Secretaría de Educación Pública a la Legación de México en Argentina, 10 de octubre, 1923, Archivo Histórico de la Secretaría de Relaciones Exteriores Genaro Estrada (en adelante AHGE), Fondo Legación de México en Argentina, leg. 21, exp. 5, f. 8. 
Antes de concluir esta breve introducción es relevante mencionar que la búsqueda por establecer un sistema de intercambio fue parte de un proceso mucho más amplio, que involucró a diversos actores, desde sociedades privadas de filantropía hasta partidos políticos, pasando por organismos internacionales o religiosos y proyectos culturales de largo alcance. ${ }^{10}$ Además, cada acción significaba necesariamente el establecimiento de reciprocidades. De alguna manera, según las bases de la diplomacia de aquel momento, cada ofrecimiento debía ser retribuido equitativamente. Esto involucró relaciones equilibradas con algunos países, pero también marcó dependencias o preeminencias sobre otros. ${ }^{11}$

\section{UN ESTUDIO PENDIENTE}

En la historiografía, el tema de la implementación del sistema de becas, también llamadas pensiones, ha sido escasamente trabajado, incluso, entre quienes se dedican a investigar acerca de los estudiantes o las universidades. ${ }^{12}$ Esto me parece particularmente interesante, especialmente si tenemos en consideración la

${ }^{10}$ Por ejemplo, el accionar de las Conferencias Panamericanas fue un elemento clave. Este proceso estuvo asociado a la búsqueda de unir el continente americano en torno a Estados Unidos, e incluyó la creación de becas para estudiantes latinoamericanos, como la Guggenheim, entre otras. Por su parte, España, en un intento por reactivar cierto hispanoamericanismo, también ofreció apoyo a los estudiantes del otro lado del Atlántico. Véase Walter C. Thurston, "Relations with Our Latin American Neighbors", en Annals of the American Academy of Political and Social Science, vol. 156, julio de 1931, pp. 116-125; "The Guggenheim Foundation: Announcement of Preliminary Gift of Three Million Dollars to Establish John Simon Guggenheim Memorial Foundation Endowing Fellowships for Advanced Study Abroad", en The High School Journal, vol. 8, núms. 3/5, University of North Carolina Press, marzo-mayo, 1925, pp. 48-50; Aimer Granados, "La corriente cultural de la Jae en México: el Instituto Hispano Mexicano de Intercambio Universitario, 1925-1931", en Revista de Indias, vol. LXVII, núm. 239, Instituto de Historia (CSIC), 2007, pp. 103-124.

${ }^{11}$ Jürgen Buchenau, In the Shadow of the Giant. The Making of Mexico's Central America Policy, 1876-1930, Alabama, The University of Alabama Press, 1996.

12 Véanse los tres tomos coordinados por Renate Marsiske, Movimientos estudiantiles en la bistoria de América Latina, México, Centro de Estudios sobre la Universidad-unam/Plaza y Valdés, 1999. 
importancia que posee actualmente el intercambio académico dentro de las relaciones exteriores mexicanas.

Ahora bien, en este sentido el trabajo de Aimer Granados es quizá uno de los pocos que intenta introducirse en el mundo de la cooperación cultural desde esta perspectiva. Su texto analiza el rol que tuvo el Instituto Hispano Mexicano de Intercambio Universitario (IHMiu) en la creación y consolidación de vínculos entre ambas naciones entre 1925 y $1931 .{ }^{13}$ Si bien es un artículo que estudia particularmente el desempeño de esta institución, sin referirse al contexto general de multiplicación de intercambio académico, muchos de los nombres citados por Granados son trascendentales para pensar las relaciones de México con el resto del continente americano, como es el caso de Pedro Henríquez Ureña, Alfonso Reyes o Daniel Cossío Villegas. En el caso del IHмiu, el intercambio intelectual estuvo relacionado con la idea de una "inmigración privilegiada", o en otras palabras, los españoles son bienvenidos porque poseen un "capital cultural" que los mexicanos pueden aprovechar. ${ }^{14}$ Sin embargo, hay un desequilibrio en la relación, marcada por un renacer del hispanoamericanismo, que se encuentra en el centro de las iniciativas educativas impulsadas por José Vasconcelos y los ateneístas. ${ }^{15}$

Un tema interesante es que esta institución actuó no sólo en México, sino que fue capaz de insertarse dentro de toda una red de organismos de alcance continental. Así, por ejemplo, a través de la Unión Iberoamericana logró establecer visitas periódicas de intelectuales a tierras mexicanas, para luego llevarlos a Chile, Uruguay y Argentina. Sin embargo, algo que no preocupa a Granados es que este mismo proyecto institucional fue retomado como modelo desde la Sociedad Mexicano-Americana de Intercambio Escolar (Mexican-American Scholarship Fundation), que operaba, por lo menos, desde 1922. O que incluso, en

13 Granados, op. cit.

${ }^{14}$ Sebastian Faber, Exile and Cultural Hegemony: Spanish Intellectuals in Mexico (1939-1975), Nashville, Vanderbilt University Press, 2002; Clara Lida, "El perfil de una inmigración: 1821-1939", en Clara Lida [comp.], Una inmigración privilegiada. Comerciantes, empresarios y profesionales españoles en México en los siglos XIX y XX, Madrid, Alianza Editorial, 1994, pp. 25-51.

${ }^{15}$ Granados, op. cit. 
1934 el ministro de asuntos extranjeros de Francia, Paul Bargeton, informaba al embajador mexicano en París que estaba todo preparado para poner en funcionamiento el Comité Franco-Mexicano de Intercambio Universitario. ${ }^{16}$ Este organismo tendría como objetivo estrechar los vínculos culturales entre ambos países, haciendo una especial mención a la presencia permanente de estudiantes mexicanos en las universidades francesas. ${ }^{17}$

Otro texto que se refiere directamente al intercambio académico es el publicado por Mílada Bazant en la revista Historia Mexicana, relativo a los estudios en el extranjero de los hermanos Urquidi. ${ }^{18}$ Este texto intenta de alguna manera entregar un marco de análisis para una multiplicidad de experiencias de estudiantes en el extranjero, que al parecer constituía parte ineludible dentro del proceso formativo de la élite porfiriana. El seguimiento de estos hermanos en sus lugares de estudio nos recuerda la importancia de matizar el tratamiento histórico estructural con las vivencias propias de los sujetos. Pese a ello, me parece que en el texto de Bazant hay una carencia de relaciones entre los procesos personales y las condiciones políticas o sociales, una falta de diálogo entre lo particular y lo general. De todas maneras, lo relevante de este artículo es que nos permite comprender que la formación de un "sistema" estatal de intercambio académico fue un proceso de largo plazo, con muchos vaivenes y donde los intereses de los actores fueron fundamentales. Sin el ímpetu de los becarios, muchos de los intercambios nunca se hubieran podido desarrollar.

Un tercer trabajo que se ocupa de las "becas" es el desarrollado por Lucila López, concentrándose en aquellas entregadas por la Junta Cívica en 1825 para

${ }^{16}$ Carta de Henri Goiran, de la Legación de la República Francesa en México, a Ezequiel A. Chávez, presidente del Comité Franco-Mexicano de Intercambio Universitario, 9 de julio, 1934, Archivo Histórico unam (en adelante AHunam), Fondo Ezequiel A. Chávez, sección universidad, caja 8 , doc. 83.

17 Véase Arturo Taracena Arriola, "Latin Americans in Paris in the 1920s: the Anti-imperialist Struggle of the General Association of Latin American Students, 1925-1933", en Ingrid Fey y Karen Racine [eds.], Strange Pilgrimages. Exile, Travel and National Identity in Latin America, 18001990s, Wilmington, Jaguar Books, 2000, pp. 131-146.

${ }^{18}$ Mílada Bazant, "Estudiantes mexicanos en el extranjero: el caso de los hermanos Urquidi", en Historia Mexicana, vol. 36, núm. 144, El Colegio de México, 1987, pp. 739-758. 
celebrar el XV Aniversario de la Independencia de México. ${ }^{19} \mathrm{Si}$ bien es un texto que se refiere a un periodo lejano a los intereses de esta investigación, entrega varios elementos que se problematizarán en las siguientes páginas. En primer lugar, la autora es muy precisa en vincular la entrega de estos apoyos con la búsqueda de objetivos de tipo político cultural. En segundo término, considera las becas como figuras centrales de una "arquitectura" de la celebración. Y por último, destaca la tensión existente entre los preparativos apoteósicos del festejo y la tremenda debilidad financiera del Estado mexicano posterior al proceso independentista. Veremos a continuación cómo estos elementos, en conjunción con algunos otros, se combinan en el sistema de becas que se intentó gestar en el México posrevolucionario.

\section{El aÑo Clave, 1922}

Las primeras pensiones que otorgó el gobierno mexicano a extranjeros para que estudiaran en el país fueron establecidas por Álvaro Obregón a fines de 1921 y puestas en marcha en $1922 .{ }^{20}$ El proceso comenzó desde una de las cuestiones más sensibles para el nuevo gobierno: el 17 de noviembre de 1921, la Secretaría de Guerra anunció que concedía quince becas para el Colegio Militar y cinco para la Escuela de Aviación. Estaban destinadas fundamentalmente a centroamericanos y a estudiantes de los ejércitos del Cono Sur. ${ }^{21}$

19 Lucila López de la Vega, "Las celebraciones de 1825: del patriotismo a la educación. Las becas educativas de la Junta Cívica", en Historia Mexicana, vol. 60, núm. 237, El Colegio de México, 2010, pp. 641-655.

${ }^{20}$ Coincidentemente ese año se celebró el Centenario de la Independencia, con la realización de un Congreso Internacional de Estudiantes. Véase Roberto Machuca Becerra, América Latina y el Primer Congreso Internacional de Estudiantes de 1921, México, 1996 (Tesis de licenciatura en Estudios Latinoamericanos, FFyL-UnAM), 184 pp.

${ }^{21}$ Estas becas fueron ofrecidas desde las legaciones de Argentina, Chile y Uruguay. Pero se supeditaron a que dichos países ofrecieran un trato equivalente. Desconozco la respuesta de Uruguay y Chile (aunque este país mantuvo un fluido intercambio castrense con México durante este periodo). Argentina por su parte aceptó desarrollar dicho intercambio. Véase Me- 
Casi inmediatamente, por recomendación de Juan de Dios Bojórquez, ministro plenipotenciario de México en Centroamérica, Obregón decidió entregar un total de 60 becas a estudiantes de aquella zona. Se repartirían 12 por cada país: Guatemala, El Salvador, Honduras, Nicaragua y Costa Rica. Panamá quedaría fuera de este ofrecimiento. ${ }^{22}$ Como explica María del Carmen Díaz: "Bojórquez consideraba que por medio de los estudiantes, se conseguiría mayor acercamiento con los pueblos centroamericanos y que algunos profesionistas eran tan mexicanos como nosotros y hacían la mejor propaganda de México en el extranjero. Esta idea agradó a Obregón" ${ }^{23}$ Y por supuesto coincidía con los planes desarrollados por el entonces secretario de Educación Pública, José Vasconcelos, aunque hubiera preferido extender el ofrecimiento más allá del istmo. ${ }^{24}$

Años más tarde, Plutarco Sánchez Herrera, uno de los beneficiados costarricenses, describió el proceso:

[... e en el año de 1922, siendo presidente de la república el general Álvaro Obregón, comprendiendo aquel gobernante generoso que era indispensable crear vínculos de hondo afecto entre todos los pueblos de nuestra América y deseando

morándum de la Legación de México en Argentina, 30 de noviembre, 1923, AHGE, Fondo Legación de México en Argentina, leg. 21, exp. 4.

22 "Apuntes biográficos y breves consideraciones sobre el General Obregón", en Acción Social, 18 de julio, 1931, p. 9.

${ }^{23}$ María del Carmen Díaz Vázquez, "Centroamérica en la estrategia diplomática mexicana: entre la política y la difusión cultural (1920-1932)", en Diálogos Revista Electrónica de Historia, núm. especial, 2008. En http:/historia.fcs.ucr.ac.cr/dialogos.htm. También podría leerse como una manera de contrarrestar el espacio que Estados Unidos comenzaba a ocupar en el intercambio académico, reemplazando a Francia como principal foco de atracción. Véase Bryce Wood, "Scholarly Exchanges between Latin America and the United States", en Proceedings of the Academy of Political Science, vol. 30, núm.4, Columbia University, 1972, pp. 123-140.

${ }^{24}$ Fell, op. cit. De todas maneras, se desarrollaron algunos intentos aislados por extender el intercambio a otros países. Véase la correspondencia entre Ezequiel A. Chávez, quien se desempeñaba como rector de la Universidad Nacional de México, y Lourival de Guillobel, secretario Encargado de Negocios ad ínterim de Brasil en México, donde se propone el intercambio académico entre ambos países. Carta de Ezequiel A. Chávez, rector de la Universidad Nacional de México, a Lourival de Guillobel, secretario Encargado de Negocios ad ínterim de Brasil en México, 27 de noviembre, 1924, AHUnAm, Fondo Ezequiel A. Chávez, sección universidad, caja 8, doc. 42. También la propuesta de Gabriela Mistral, "Sobre el intercambio universitario", en El Mercurio de Santiago, 11 de mayo de 1924, p. 8. 
hubiera una más íntima y mutua comprensión entre México y los países de Centro América y convencido de que es en el corazón de la juventud donde mejor germina todo noble y alto sentimiento, tuvo a bien conceder unas becas a un grupo de estudiantes centroamericanos para que vinieran a hacer sus estudios profesionales a diversas escuelas de esta república. ${ }^{25}$

Por su parte, el escritor hondureño Rafael Heliodoro Valle, vinculado laboralmente a la SEP, felicitó públicamente a las autoridades mexicanas por el empeño puesto en el intercambio estudiantil: "Desde Honduras, con el agrado y el aplauso del pueblo hondureño, pronto partirían de Amapala hacia la capital de México once becarios de la Secretaría de Educación Pública (uno de ellos ya se encuentra allá) y cuatro pensionados por la Secretaría de Guerra". ${ }^{26}$

Ahora bien, para el gobierno mexicano pasar de las palabras a las acciones concretas involucró sortear una serie de desafíos. Las primeras preguntas importantes que surgieron fue a quién asignar las becas, y mediante qué mecanismos. Obviamente las respuestas a estas preguntas obedecían a un entramado tanto político diplomático como educativo cultural. Por ejemplo, el mismo Bojórquez, quien también actuaba como delegado de la Universidad Nacional en Centroamérica, consideraba que el gobierno mexicano cometía un error al ordenar que las becas se concedieran por conducto de los gobiernos, porque "en vez de beneficiar a una nación, convierte el valioso obsequio en botín político, que el gobierno de El Salvador y Nicaragua emplearán para comprometer a sus enemigos, o fortalecer alianzas con morbosos elementos de sus carcomidas administraciones". ${ }^{27}$

${ }^{25}$ Carta de Plutarco Sánchez Herrera al secretario de Educación, 2 de enero, 1929, AHSEP, Fondo Departamento Administrativo, caja 12, exp. 5, f. 78.

${ }^{26}$ Carta de Rafael Heliodoro Valle dirigida a Bojórquez, México, 8 de marzo, 1922. Expediente de Juan de Dios Bojórquez, Fondo Reservado Rafael Heliodoro Valle (en adelante FrHv), Biblioteca Nacional de México.

${ }^{27}$ Carta de Juan de Dios Bojórquez al secretario de Educación, sin fecha, AHGE, Expediente personal de Juan de Dios Bojórquez, expediente 1-131-333, parte I. Para comprender las diferentes posturas al respecto de este debate conviene revisar el panorama general que dibuja Jürgen Buchenau sobre la política mexicana en Centroamérica. Véase Jürgen Buchenau, In the shadow of the Giant. The Making of Mexico's Central America Policy, 1876-1930, Alabama, The University of Alabama Press, 1996, especialmente el capítulo 6. 
En definitiva, la manera de elegir a los beneficiados fue una mezcla de todas las posibilidades, desde la elección presidencial hasta la votación entre los mismos estudiantes. En el expediente de Jesús Posada es donde mejor se evidencian estos múltiples mecanismos. En 1923 el Consulado de El Salvador en México envió una solicitud a la Secretaría de Educación Pública para llenar el lugar del estudiante Abelardo Rodríguez, que había renunciado a su beca. Escribe el cónsul, Constantino Hernández: "he recibido instrucciones del señor Presidente Constitucional del El Salvador, recomendándome muy especialmente al joven Jesús Posada". ${ }^{28}$ La respuesta de las autoridades mexicanas reconoce que en algún momento, provisionalmente, Abelardo Rodríguez estuvo becado, porque cuando fue la convocatoria se encontraba en México, pero el auténtico beneficiado de la beca llegó en julio de 1922, Félix González, trayendo las credenciales que lo acreditaban como "alumno electo en plebiscito". ${ }^{29}$ Hasta aquí evidenciamos tres mecanismos distintos de acceder a las becas, recomendación presidencial, por haber estado ya estudiando al momento de la convocatoria y por elección estudiantil en el propio país. ${ }^{30}$ Veamos ahora una cuarta manera.

En octubre de 1923, la venezolana Josefina Juliac, envió una carta a José Vasconcelos pidiéndole una beca para su hermano menor:

Yo, que no puedo resignarme a ver a mi hermano, inteligente, aspirando a su desenvolvimiento intelectual, convertirse en un número más en esta máquina de obreros neoyorquinos, he pensado, Doctor, que tal vez a Ud. le sería fácil concederle una beca en la Universidad de México, proporcionándole al mismo tiempo un

${ }^{28}$ Constantino Hernández, cónsul de México en El Salvador al secretario de Educación, 24 de julio, 1923, AHSEP, Fondo Departamento Administrativo, caja 6, exp. 36, f. 1.

${ }^{29}$ Carta del director de la Escuela Superior de Comercio y Administración al secretario de Educación, 26 de julio, 1923, AHSEP, Fondo Departamento Administrativo, caja 6, exp. 36, f. 2. En el caso de Costa Rica la adjudicación de las becas fue confiada a una Junta integrada por el personal de la Legación de México y los directores de los colegios de educación secundaria y de la Biblioteca Nacional. Expediente de Rigoberto Álvarez Berrocal, AHSEP, Fondo Departamento Administrativo, caja 6, exp. 48, f. 53.

${ }^{30}$ Las becas para los salvadoreños se distribuyeron de la siguiente manera: dos para el Colegio Militar; dos para la Escuela Nacional de Agricultura; dos para la Escuela Nacional de Industrias Químicas; dos para la Escuela Práctica de Ingenieros Mecánicos; dos para la Escuela de Bellas 
trabajo de modesta remuneración que le permita sostenerse durante el tiempo de sus estudios. ${ }^{31}$

Antes de la petición, la sudamericana había realizado un extenso comentario de sus acciones en contra del dictador venezolano Juan Vicente Gómez, un tema que para el ministro de educación era bastante cercano (al igual que el rechazo a Estados Unidos). ${ }^{32}$ Vasconcelos no sólo le otorgó la beca al hermano, sino que lo hizo quitándole un lugar a Nicaragua y ofreciéndole además 150 dólares para cubrir los gastos de transporte. Es destacable que en toda la carta de petición y en la respuesta del secretario no se mencione el nombre del hermano de la solicitante, Pedro Alberto Juliac. Fue el único venezolano y sudamericano beneficiado con estas becas.

La irregularidad en la entrega de estos beneficios es una discusión que acompañará prácticamente todo el proceso.

\section{¿COYUNTURAS O PLANIFICACIÓN?}

Pero los problemas no acababan con la elección de los becarios, el siguiente paso conflictivo, antes de que comenzaran sus clases, fue traerlos a México. Algo que hoy en día no representaría mayores dificultades, para aquella fecha, con un sistema de transporte poco desarrollado y costoso, se transformó en un verdadero desafío. Una de las primeras acciones fue conseguir que los ferrocarriles mexicanos hicieran descuentos a los estudiantes. ${ }^{33}$ Incluso, algunos padres $\mathrm{u}$

Artes y Conservatorio de Música; y dos para las facultades de la Universidad. Expediente de Gonzalo Félix, AHSEP, Fondo Departamento Administrativo, caja 6, exp. 1, f. 1.

${ }^{31}$ Carta de Josefina Juliac a José Vasconcelos, 19 de octubre, 1923, AHSEP, Fondo Departamento Administrativo, caja 6, exp. 10, f. 2.

32 Brian S. McBeth, "Foreign support for Venezuelan political exiles during the regime of Juan Vicente Gómez: the case of Mexico, 1923-1933", en The Historian, Phi Alpha Theta History Honor Society, 2007.

33 Véase la revista El Libro y El Pueblo (ELYEP), t. I, núm. 12, febrero de 1923. En este número Bojórquez reflexiona sobre su labor en América Central durante el año 1922. "Tengo el gusto de anunciar a usted que mi último trabajo de positivo acercamiento entre aquellos estudiantes [...] 
apoderados aprehensivos pedían acompañar a los hijos, por lo menos en una parte del trayecto. ${ }^{34}$

El 22 de mayo de 1922, Aarón Sáenz, subsecretario de la Secretaría de Relaciones Exteriores, envió la siguiente información a la Secretaría de Educación Pública: "Nuestra legación en San Salvador comunica que Héctor Escobar y once estudiantes salvadoreños más, llegarán a Mariscal, [el]veintisiete [del] actual [mes] aproximadamente. Rogando a Ud. dar órdenes de pasajes hasta México de estación aludida". ${ }^{35} \mathrm{Al}$ día siguiente se anunciaba que los bequistas [sic] salvadoreños partirían el 24 vía Suchiate. El 27 de mayo las informaciones traían problemas, los pensionados quedaron detenidos en Ayutla, Guatemala, porque el ferrocarril no había recibido la autorización para llevarlos. ${ }^{36}$ En estas condiciones, el movimiento de los estudiantes no pasó desapercibido, los diarios informaron de ellos, también las agencias de noticias y los encargados diplomáticos, incluso el mundo del espionaje no les perdía de vista. ${ }^{37}$

Estos problemas de transporte no tenían la conexión política que se evidenció en la distribución de las becas. Al contrario, parecen tener un cariz marcadamente financiero. Sin embargo, los gastos en los que incurría el Estado se transformaron rápidamente en un tema muy delicado. De hecho, hubo un abierto cuestionamiento de ciertos sectores políticos al gobierno de Álvaro Obregón por preferir financiar a extranjeros frente a mexicanos. Problema que afectó

es el de haber logrado que aquellos Ferrocarriles Nacionales ofrezcan la reducción de un 50\% de los pasajes de los estudiantes que viajan en dicha línea hacia nuestro país", p. 184.

${ }^{34}$ Carta de Henaida Alcazar al secretario de Educación, 5 de enero, 1929, AHSEP, Fondo Departamento Administrativo, caja 12, exp. 10.

35 Memorándum de Aarón Sáenz, subsecretario de la Secretaría de Relaciones Exteriores, al secretario de Educación, 22 de mayo, 1922, AHSEP, Fondo Departamento Administrativo, caja 6, exp. 36, f. 8 .

${ }^{36}$ Memorándum de Aarón Sáenz, subsecretario de la Secretaría de Relaciones Exteriores, al secretario de Educación, 27 de mayo, 1922, AHSEP, Fondo Departamento Administrativo, caja 6, exp. 36, f. 10.

37 "The latin american students have started to arrive in Mexico City to pursue the technical studies in the principal educational centers of the city. These are the men who were granted scholarships by the Mexican Government", informaba el agregado militar de Estados Unidos a su gobierno. FaPECFT, Fondo CDEeum (Espías), exp. 070101, inv. 23, leg. 2/11. 
tanto a los becarios centroamericanos, como posteriormente al peruano Víctor Raúl Haya de la Torre o a la poetisa chilena, Gabriela Mistral, entre otros. ${ }^{38}$ De hecho, en 1922 el presidente de México, a través de José Vasconcelos, también debió entregar siete becas a la Federación de Estudiantes Mexicanos (FEM). ${ }^{39}$ Éstas podían ser utilizadas en algún país de América Latina. La irregularidad de la entrega de becas para que fueran gestionadas por los estudiantes y no por la Universidad, según Ricardo Melgar Bao, se puede leer como un signo de acercamiento entre los estudiantes y el gobierno. ${ }^{40} \mathrm{La}$ organización estudiantil en abril de ese año había renunciado a los objetivos que había trazado el Congreso Internacional de Estudiantes de 1921. Abandonaba la radicalidad de aquel programa, y como moneda de canje recibía entre otras cosas, un ramillete de becas en el extranjero.

Pero esta tensión entre un país que se mostraba generoso y la debilidad financiera del gobierno, no puede pasar desapercibida y debe continuar siendo explorada. De hecho, el Departamento de Intercambio Universitario reconocía ese mismo año que "[...] ha decidido posponer las labores relativas para cuando sea posible distraer hacia ellas la atención y el dinero que ahora reclaman asuntos de mayor urgencia para el pueblo mexicano". 41

También en este mismo periodo se creó el primer Reglamento de Pensiones Universitarias, que disponía que: "Para la tercera clase [pensionados extranjeros en México] un 5\% de las pensiones que se concedan se destinarán a la facultad de jurisprudencia; a medicina y odontología un 15\%; a la facultad de

38 Ricardo Melgar Bao, "Redes del exilio aprista en México (1923-1924): una aproximación", en Pablo Yankelevich [coord.], México, país refugio. La experiencia de los exilios en el siglo XX, México, INAH/Plaza y Valdés, 2002.

39 Fell, op. cit.

${ }^{40}$ Ricardo Melgar Bao, Redes e imaginario del exilio en México y América Latina, 1934-1940, Buenos Aires, Ediciones Libros en Red, 2003.

${ }^{41}$ Departamento de Intercambio Universitario, "Intercambio de alumnos y de profesores", en Boletín de la Secretaría de Educación Pública, t. I, núm. 2, 1º de septiembre de 1922, p. 138. De todas maneras había dispuesto 15 mil pesos para el intercambio académico vinculado a la Escuela de Verano de la Universidad Nacional. Desde 1925 el gobierno mexicano ofrecía sufragar los costos de dos representantes para cada país centroamericano y del Caribe. 
ingenieros un 30\%; a la de química un 30\%, y a la de Altos Estudios un 20\%”. 22 En la redacción de este sucinto documento participaron Vicente Lombardo Toledano, Daniel Cossío Villegas y el doctor Pedro de Alba. Un representante de la Secretaría de Educación Pública, uno de los estudiantes y un tercero de la Universidad, respectivamente.

Pero los reglamentos no fueron suficientes y cuando los estudiantes centroamericanos llegaron a México, las autoridades debieron hacerse cargo de desarrollar de la mejor forma posible los compromisos adquiridos. Dentro de este proceso, y según los expedientes de los becarios, el mes de mayo de 1924 parece ser clave en la planificación estatal de las pensiones. En ese periodo todos los becarios debieron llenar una pequeña ficha con sus datos básicos, nombre, edad, lugar de procedencia, adjuntar un par de fotografías, además de mencionar cuánto tiempo les quedaba para terminar sus estudios. ${ }^{43}$ Esta última información al parecer, y según los problemas que tiene el costarricense Rigoberto Álvarez Berrocal por no anotarla, es la más importante para las autoridades.$^{44} \mathrm{El}$ inconveniente que debían enfrentar en aquel entonces era uniformar y tener certeza del avance de cada uno de los becarios. Muchos de los recién llegados habían cambiado de carrera, ${ }^{45}$ otros debieron terminar la escuela preparatoria antes de entrar a la Universidad. Incluso, los lugares de quienes decidieron renunciar fueron llenados a posteriori. ${ }^{46}$ La disparidad no sólo involucraba pro-

${ }^{42}$ Departamento de Intercambio Universitario, "Intercambio de alumnos y de profesores", en Boletín de la Secretaría de Educación Pública, t. I, núm. 2, 1º de septiembre de 1922, p. 138. En el siguiente reglamento, elaborado en 1925, estas disposiciones fueron eliminadas.

43 Este proceso de filiación se repitió en 1926.

${ }^{44}$ Expediente de Rigoberto Álvarez Berrocal, AHSEP, Fondo Departamento Administrativo, caja 6, exp. 48.

45 De los expedientes revisados el caso más interesente es el de Jesús Posada, quien recibe la beca para estudiar Química Industrial, luego pasa a la Escuela Nacional Preparatoria, continúa estudiando Auxiliar de Farmacia, luego pide cambiarse a la Escuela de Ingenieros Mecánicos y Electricistas (donde sólo toma dos materias), y antes de que le quiten la beca decide ir a la Escuela de Química de Tacuba. Expediente de Jesús Posada, AHSEP, Fondo Departamento Administrativo, caja 6, exp. 36.

${ }^{46}$ En el expediente incompleto del hondureño Humberto Velásquez la última renovación que consta de su beca es el año 1930. Había iniciado en 1922, por lo tanto, llevaba 8 años recibiendo 
blemas de orden administrativo, como por ejemplo recibir los informes de asistencia y de calificaciones, sino que también complicaba el ejercicio financiero de la Secretaría. Como ya podemos percibir, éste era uno de los temas centrales al momento de referirse a los becarios centroamericanos, tanto en los expedientes de cada uno de los pensionados, como en las memorias y boletines de la propia dependencia. ${ }^{47}$ Por el contrario, los logros educativos, los aportes intelectuales, los avances en la integración latinoamericana son temáticas ausentes en los informes públicos.

De todas maneras, los desafíos que debieron enfrentar los becarios tuvieron relación con el proceso constitutivo del sistema educativo mexicano posrevolucionario. ${ }^{48}$ Los cambios que enfrentó la organización educacional en el país en muchos casos significaron que los pensionados quedaran a la deriva, mientras se implementaban las modificaciones. Por ejemplo, en 1925 se clausuró indefinidamente el Colegio Militar, y los becarios centroamericanos Héctor René Morales, Luis Humberto Días, Rafael Ángel Freer y Humberto Velásquez, quedaron varios meses sin saber qué hacer. La respuesta de las autoridades mexicanas fue simplemente avisar a las embajadas respectivas, mientras los estudiantes dudaban entre mantenerse y trabajar en México o volver a su patria esperando que reabrieran el centro formativo. ${ }^{49}$ Para inicios de 1926 esta condición se había normalizado y el Colegio Militar reinició sus clases. Pero esta situación no fue aislada, afectó tanto a estudiantes de la Escuela Nacional

el subsidio del gobierno mexicano. Expediente de Humberto Velásquez, AHSEP, Fondo Departamento Administrativo, caja 12, exp. 9.

47 Véase Secretaría de Educación Pública, Noticia estadística sobre la Educación Pública en México, correspondiente al año de 1926, México, 1928, pp. 354 y 355. Es una tabla con los nombres de los becarios y el gasto de la SEP por cada uno.

48 Por ejemplo, entre 1924 y 1928 el rector Alfonso Pruneda, correspondiendo al afán centralizador del gobierno de Calles, había tratado de unir las distintas escuelas nacionales que había sido la Universidad hasta entonces. Véase Renate Marsiske, "Crónica del movimiento estudiantil de México en 1929", en Revista Historia de la Educación Latinoamericana, núm.1, Universidad Pedagógica y Tecnológica de Colombia, 1998, pp. 35-62.

49 Expediente de Humberto Velásquez, AHSEP, Fondo Departamento Administrativo, caja 12, exp. 9. Humberto Velásquez incluso solicitó a la SEP financiamiento para regresar a Honduras mientras duraba la clausura. 
Preparatoria, a quienes cursaban en la Universidad Nacional de México, y a los adscritos a la Escuela Nacional de Agricultura. ${ }^{50}$

Como ya vimos, los mecanismos para otorgar las becas no fueron precisos ni homogéneos, lo que generó diversos problemas tanto al gobierno como a las instituciones receptoras. Los encargados de la SEP en 1922 reconocían que "[... el intercambio de alumnos y de profesores con Universidades extranjeras requiere de minuciosos trabajos previos de adaptación (pues entre México y los países extranjeros hay gran disparidad en planes de estudios, programas, métodos y hasta en la duración del año escolar y la retribución del profesorado)" ${ }^{51}$ Una de las complicaciones más evidentes fue la idoneidad académica de los becarios y, si algo remarcaba la SEP en su informe de 1928, era que los estudiantes recibidos no habían satisfecho el rendimiento escolar mínimo que se esperaba de ellos. ${ }^{52}$ Muchos fueron reprobados en las carreras que habían elegido, otros simplemente tuvieron un desempeño que bordeaba la línea mínima de aprobación. En el caso del salvadoreño Francisco Osegueda, inicialmente inscrito en la Facultad de Medicina, fue devuelto a continuar sus estudios en la Escuela Nacional Preparatoria. Había reprobado todas las materias que cursó. ${ }^{33}$ Pedro Alberto Juliac, de nacionalidad venezolana, fue más sincero y antes de comenzar los cursos en 1924, pidió no ser enviado aún a la Facultad de Medicina. Su hermana, al momento de solicitar su beca, "[...] cometió el error de no indicar que todavía yo no había terminado los estudios preparatorios". ${ }^{4}$

${ }^{50}$ En algunos casos, los estudiantes decidieron participar de los conflictos, a pesar de las posibilidades de expulsión de la universidad e, incluso, de la aplicación del Artículo 33 Constitucional, que permite la expulsión de los extranjeros que participen de la política nacional. Véase AHSEP, Fondo Departamento Administrativo, caja 6, exp. 45 y exp. 3. En ambos ejemplos los estudiantes fueron expulsados de sus centros educativos, aunque gracias a posteriores gestiones de la SEP, fueron reincorporados.

51 "Intercambio de alumnos y de profesores", en Boletín de la Secretaría de Educación Pública, t. I, núm. 2, $1^{\circ}$ de septiembre de 1922, p. 138.

52 Véase el Cuadro 2.

53 Expediente de Francisco Osegueda, AHSEP, Fondo Departamento Administrativo, caja 6, exp. 33.

${ }^{54}$ Carta de Pedro Juliac a José Vasconcelos, 2 de mayo, 1924, AHSEP, Fondo Departamento Administrativo, caja 6, exp. 10, f. 20. Véase también AHunam, Fondo expedientes alumnos, cuenta 27032, exp. Pedro Alberto Juliac. 
Frente a todos estos problemas los recién llegados no se mantuvieron inmóviles. Antonio Adolfo Ramírez, salvadoreño, junto a Plutarco Sánchez Herrera, enviaron una carta, en febrero de 1923 al Departamento Administrativo de la SEP en la que exponían sus desavenencias con la Escuela Nacional de Agricultura. Cuestionaban en primer lugar el régimen militarizado de la institución, pero "[...] preferimos pasar por alto este punto, para exponerle otro de los motivos que hacen detestable el internado. Este otro motivo señor, es la pésima comida que allí se nos daba, lo que hacía que todo el día estuviésemos soñolientos y faltos de esa energía natural en la juventud y tan necesaria en el estudiante". ${ }^{55}$ Por estos motivos, ambos estudiantes solicitaban poder inscribirse como alumnos externos, algo que las autoridades de la sEP les permitieron. Sánchez se verá posteriormente envuelto en las movilizaciones estudiantiles en contra de las autoridades y al borde de la expulsión. ${ }^{56}$

\section{DE LAS ENFERMEDADES Y CARENCIAS MATERIALES}

Entre los problemas que debieron enfrentar los becarios centroamericanos en México, no sólo encontramos las añoranzas de la tierra natal o la melancolía provocada por estar lejos de la familia. Muchas veces las pensiones apenas alcanzaban para cubrir los gastos básicos y muchos de ellos debieron realizar trabajos fuera del ámbito académico. ${ }^{57}$ El 17 de noviembre de 1925, el Departamento Administrativo de la SEP le mandó un memorándum al costarricense Plutarco Sánchez Herrera, preguntándole cómo piensa pagar una deuda de 300 pesos que tiene con la Escuela Nacional de Agricultura. El estudiante responde que:

55 Carta de Plutarco Sánchez Herrera y Antonio Adolfo Ramírez al jefe del Departamento Administrativo de la SEP, 12 de febrero, 1923, AHSEP, Fondo Departamento Administrativo, caja 6, exp. 36, f. 8 .

${ }^{56}$ Expediente Plutarco Sánchez Herrera, AHSEP, Fondo Departamento Administrativo, caja 6, exp. 45.

${ }^{57}$ Una situación similar afectaba a los estudiantes mexicanos en el extranjero descritos por Bazant, op. cit. 
El adeudo que he contraído con esta escuela, lo cancelaré con lo que perciba de las pensiones correspondientes a los meses de noviembre y diciembre del presente año. Los meses de enero y febrero del próximo año los pasaré en una Hacienda, a la cual me envía la Dirección de esta Escuela, en vía de práctica, y esta misma dirección me ha concedido, que cancele el resto de mi adeudo con lo que perciba en esos meses. ${ }^{58}$

Muchos de los expedientes de los becarios demuestran que los 80 pesos de pensión mensual que se les destinaron en 1922 (y los 50 pesos de una sola vez, para su instalación), alcanzaban en el mejor de los casos para la subsistencia. Algunos ahorraron un poco viviendo en la Casa Nacional del Estudiante, de origen porfiriano; otros prefirieron compartir alguna habitación en las cercanías de su lugar de estudio; la mayoría evitaba realizar gastos que no fueran los rigurosamente necesarios para desarrollar sus estudios. De todas maneras, cualquier situación que alterara el curso de la vida cotidiana de los estudiantes, como enfermarse o tener que viajar, podía resultar muy problemática para los bolsillos de los pensionados.

Una forma de evitar o, por los menos, contrarrestar las inseguridades de la vida en México fue la inserción de los estudiantes pensionados en pequeñas comunidades de inmigrantes de su propia localidad. En la mayoría de los casos, los amigos de estos becarios eran los connacionales que se encontraban en su misma situación, otros centroamericanos con quienes compartían el espacio universitario, o incluso dentro de su misma aula. Muchos de los poderes notariales que dejaron los pensionados para que alguien más pasara por la pagaduría a cobrar su dinero, fueron entregados a compañeros en su misma situación..$^{9}$

${ }^{58}$ Memorándum del jefe del Departamento Administrativo de la sep a Plutarco Sánchez Herrera, 17 de noviembre, 1925, AHSEP, Fondo Departamento Administrativo, caja 12, exp. 5, f. 24.

59 Francisco Osegueda, salvadoreño, de la Facultad de Medicina, le entregó un poder a Héctor Escobar Serrano, otro salvadoreño, pero que estudiaba en la Facultad de Jurisprudencia. A su vez, compartía casa con Antonio Dubón también salvadoreño en la 4ta calle de Dinamarca núm. 64. AHSEP, Fondo Departamento Administrativo, caja 12, exp. 9 y exp. 35. Plutarco Sánchez Herrera, costarricense, le dio un poder a Antonio Carrillo Durán, guatemalteco, ambos compañeros de cursos en la Escuela Nacional de Agricultura. Expediente de Plutarco Sánchez Herrera, AHSEP, Fondo Departamento Administrativo, caja 12, exp. 5. Luego, el salvadoreño Antonio Adolfo Ra- 
Por supuesto, estas relaciones entre "compatriotas" no estuvieron exentas de problemas y en más de alguna ocasión los pleitos llegaron incluso a tribunales. ${ }^{60}$

Ahora bien, las relaciones de los centroamericanos, un tanto endogámicas, nos permiten cuestionarnos por la capacidad de integración que tuvieron los becados o por el recibimiento que les dieron sus pares mexicanos. Por supuesto, algunos se integraron en el México posrevolucionario, asimilaron sus costumbres, e incluso terminaron casándose en estas tierras. Pero la misma Secretaría de Educación Pública se quejaba en 1928 de la falta de interrelación que existió entre los visitantes y quienes los recibían. ${ }^{61}$

Otro problema que se puede ver en los expedientes de los pensionados es cierta tendencia a enfermarse o por lo menos a utilizar esta excusa para justificar sus inasistencias y problemas con los exámenes. "A consecuencia de una infección intestinal, caí enfermo el día 9 y lo estuve por espacio de dos semanas, lo que me impidió absolutamente presentarme al referido examen de fisiología", 62 explicaba José Pacas en una carta a las autoridades de la Facultad de Medicina. En otro caso, en el mes de junio de 1923, le fue cancelada la pensión a Rafael Antonio Rivas, "con motivo de haber sido atacado de enajenación mental y regresado a su país, quedando, por lo tanto, libre esa beca hasta ahora". 63

mírez, le entregó uno a Sánchez porque debió partir urgente a su ciudad natal. Expediente de Antonio Adolfo Ramírez, AHSEP, Fondo Departamento Administrativo, caja 6, exp. 36.

${ }^{60}$ En 1924 el Departamento Administrativo de la SEP llamó a Antonio Dubón para que resolviera un problema que había tenido con Rafael Antonio Rivas, ambos salvadoreños. No se menciona cuál fue el problema ni cómo se solucionó. Expediente de Antonio Dubón, AHSEP, Fondo Departamento Administrativo, caja 12, exp. 35. José Trabanino, salvadoreño, fue acusado de cobrar la pensión a nombre de Víctor Breenes, quien puso una demanda judicial en su contra y finalmente el gobierno mexicano decidió expulsarlo. Expediente de Víctor Breenes, AHSEP, Fondo Departamento Administrativo, caja 6, exp. 3.

${ }^{61}$ Secretaría de Educación Pública, El esfuerzo educativo en México, la obra del gobierno federal en el ramo de educación pública durante la administración del presiente Plutarco Elías Calles (1924-1928). Memoria analítico-crítica de la organización actual de la Secretaría de Educación Pública, México, SEP, s/f., p. 550.

${ }^{62}$ Carta de José Pacas a la Dirección de la Facultad de Medicina, 11 de febrero, 1924, AHSEP, Fondo Departamento Administrativo, caja 6, exp. 34, f. 15.

${ }^{63}$ Carta del jefe del Departamento Administrativo al secretario de Educación, 26 de julio, 1923, AHSEP, Fondo Departamento Administrativo, caja 6, exp. 36, f. 2. 
A su vez, quien ocupó esa pensión, Jesús Posada, estuvo convaleciente de fiebre tifoidea durante octubre y noviembre de ese mismo año. ${ }^{64}$ Quizá por esta constante, las autoridades decidieron en 1925 incluir en el nuevo reglamento de pensiones entre los requisitos para los pensionados la exigencia de tener buena salud..$^{65}$

En otras ocasiones fueron motivos familiares los que terminaron con los estudios de los pensionados. El costarricense Jorge Vega Rodríguez, estudiante de medicina de 19 años, explicaba a las autoridades de la SEP:

[...] imperiosos llamados de mi familia, reclaman mi presencia a su lado, y no pudiendo desoír sus ruegos urgentes, hago por la presente formal renuncia de la pensión a que he aludido, suplicándole de la manera más atenta, se sirva aceptarla y ordenar se me faciliten los viáticos necesarios y acostumbrados, para el regreso a mi patria, adonde iré con el recuerdo más cariñoso de México, mi segunda patria. ${ }^{66}$

Pero, isu estancia en México había sido tan significativa como para llamar a este país su segunda patria?

\section{EL LATINOAMERICANISMO}

Posiblemente, desde una mirada general, las autoridades gubernamentales hubieran respondido negativamente a dicha pregunta. A su juicio, el latinoamericanismo que había impulsado el otorgamiento de estas becas ${ }^{67}$ y había justificado el gasto financiero en momentos donde el erario público era exiguo, no fue co-

${ }^{64}$ Carta de Jesús Posada al secretario de Educación, 25 de diciembre, 1923, AHSEP, Fondo Departamento Administrativo, caja 6, exp. 36, f. 10.

65 Secretaría de Educación Pública, "Reglamento de pensiones para estudios en el país y en el extranjero", 12 de febrero de 1925.

${ }^{66}$ Carta de Jorge Vega Rodríguez al secretario de Educación, 6 de diciembre, 1924, AHSEP, Fondo Departamento Administrativo, caja 6, exp. 49, f. 23. Para ver cómo evolucionó la cantidad de becarios, véase cuadro 1.

${ }^{67}$ En el momento en que fueron otorgadas estas pensiones, en Centroamérica soplaban fuertemente los vientos del unionismo. Incluso, algunos países habían declarado su coalición en una federación. Sin embargo, ya hacia 1923 la situación había girado ostensiblemente. Buchenau, op. cit. 
rrespondido por la actuación de los centroamericanos. Las pensiones no sólo buscaron dar oportunidades académicas, sino que su gran objetivo fue consolidar la hermandad entre los países del continente y esto pareció ser la deuda más importante que los pensionados no supieron cumplir.

En la evaluación realizada por el gobierno se exponía:

[...] es penoso afirmar que, por lo general, [los becarios centroamericanos] no han correspondido al generoso deseo de proporcionarles los medios de enseñanza gratuita, persiguiendo a la vez propósitos de acercamiento espiritual con los países de su procedencia. La causa fundamental de este resultado deplorable, tiene su origen en que no siempre han sido seleccionados los candidatos por instituciones educativas oficiales o particulares de reconocido crédito, sino admitidos por medio de otros procedimientos menos adecuados. ${ }^{68}$

Nuevamente se volvía sobre la elección de los pensionados. De todas maneras, estas apreciaciones, que sirvieron para que las autoridades mexicanas replantearan todo el sistema de asignación de becas, deben ser matizadas. No sólo los ideales latinoamericanistas de los que se preciaba el gobierno deben ser relativizados, sino que también debemos cuestionar la simpleza con que se pensó que los sujetos pudieran ser "reprogramados" bajo los nuevos ritmos de la integración continental. Evidentemente los estudiantes centroamericanos por muy agradecidos que estuvieran, tenían sus propios intereses y sus propias apreciaciones políticas.

De todas maneras, los pensionados de América Central desarrollaron, si bien no con la intensidad y dirección que esperaba el gobierno mexicano, acciones y reflexiones en torno al latinoamericanismo. Muchos cambiaron sus formas de describirse, si en un principio utilizaban el nombre de su país, con el correr del tiempo los escritos que presentaban a las autoridades invariablemente comenzaban con "...el que suscribe, estudiante centroamericano". ${ }^{69}$ Otros utilizaron las palabras en boga para argumentar a su favor: "[...] porque conse-

${ }^{68}$ Secretaría de Educación Pública, El esfuerzo..., p. 550.

69 Carta de Jesús Posada al secretario de Educación (en realidad, está dirigida al ministro de Instrucción Pública), sin fecha, AHSEP, Fondo Departamento Administrativo, caja 6, exp. 36, f. 33. 
cuente con los ideales socialistas que aquí en México, en la misma escuela se me han inculcado, creí de mi deber, como socialista convencido, sacrificar en esta ocasión mi interés personal al interés supremo de todos mis compañeros [...]" ${ }^{70}$

Pero no todo fue parte del lenguaje. En 1926, Plutarco Sánchez Herrera y su compañero de la Escuela Nacional de Agricultura, Antonio Carillo Durán, de origen guatemalteco, presentaron un proyecto para realizar una gira educativa agrícola por Centroamérica, acompañados de un profesor y tres estudiantes mexicanos, enseñando en varias comunidades lo que habían aprendido durante sus años de estudio. El objetivo de fondo consistía en fortalecer la "antorcha" de la fraternidad latinoamericana. En una carta al embajador de Guatemala en México, Eduardo Aguirre Velázquez, le expresaban que:

Convencidos como estamos, de la imperiosa necesidad de fomentar por cuantos medios sea posible nuestro mutuo conocimiento, un sincero acercamiento entre México y nuestra Patria, Centro América, y comprendiendo que la labor de los estudiantes centroamericanos que actualmente disfrutamos de los favores del gobierno de esta patria para nosotros tan querida, no debe tener como finalidad única la adquisición de un título, sino, y quizá sea esto lo primordial, debe preocuparse intensamente porque desaparezca hasta lo posible ese lamentable desconocimiento mutuo que actualmente aún existe entre los diversos pueblos de nuestra América. ${ }^{71}$

En este caso la SEP aceptó que los estudiantes desarrollaran la gira y además los autorizó para disponer de sus pensiones para cubrir los gastos en que incurrieran. $^{72}$

${ }^{70}$ Carta de Plutarco Sánchez Herrera al secretario de Educación, 9 de diciembre, 1924, AHSEP, Fondo Departamento Administrativo, caja 6, exp. 45, f. 40.

${ }^{71}$ Carta de Plutarco Sánchez Herrera y Antonio Carillo Durán al embajador de Guatemala en México, Eduardo Aguirre Velázquez, 7 de diciembre, 1926, AHSEP, Fondo Departamento Administrativo, caja 12, exp. 5, f. 47.

72 Plutarco Sánchez Herrera también había solicitado participar de las misiones culturales organizadas por la Secretaría de Educación Pública, haciendo cursos de apicultura. Expediente de Plutarco Sánchez Herrera, AHSEP, Fondo Departamento Administrativo, caja 6, exp. 45. 
Otro ejemplo de los ideales latinoamericanistas es la carta de renuncia presentada por el costarricense Víctor Breenes, quien tuvo que volver a su país para curarse de una enfermedad que le impedía estudiar. Escribe:

Ya llegará el momento oportuno en que, con mi humilde pluma haga saber a mi patria, todo lo que debemos a este primoroso terruño, que nos ha acogido con verdadero amor en su seno y nos ha brindado en sus colegios la manera de beber la ciencia y el acercamiento más directo a sus hijos para quienes sentimos verdadero cariño. Es, esta la forma, en que en un futuro no muy lejano, podamos ver y sentir el hermoso ideal que tanto preocupó a nuestro gran libertador Bolívar, pues los principios más sólidos para llevar a buen fin esta noble idea, son la unión intelectual y moral de los pueblos latinos y hacia allá va México. ${ }^{73}$

No sabemos hasta dónde estos textos representan de manera fidedigna el sentir latinoamericanista de quienes los escribieron, o fueron simplemente esquemas argumentativos válidos para interactuar con las autoridades. Pero, desde otra perspectiva, podemos ver en la participación de estos egresados en sus países de origen o incluso en algunos congresos continentales posteriores, que no se alejaron demasiado de la búsqueda de la unión continental. Por ejemplo, Pedro Alberto Juliac en 1931 participó en uno de los últimos intentos de derrocar al dictador venezolano, Juan Vicente Gómez. El costarricense, Rigoberto Álvarez Berrocal se convirtió en un poeta defensor del latinoamericanismo, y mientras vivía en la sede de la Gaceta Social de México, enviaba escritos a la revista cultural Repertorio Americano. ${ }^{74}$ Héctor Escobar Serrano quien fue representante de El Salvador en el "Congreso Indigenista de Pátzcuaro", estudió en México como pensionado en la Facultad de Jurisprudencia. Unos años antes, en 1935, había sido signatario del Tratado de Confraternidad Centroamericana, donde:

Guatemala ofrece a las cuatro Repúblicas hermanas, como prenda de sus sincera fraternidad, cinco becas para cada Estado en el Instituto Nacional; cinco en la Es-

${ }^{73}$ Carta de Víctor Breenes al secretario de Educación, sin fecha, AHSEP, Fondo Departamento Administrativo, caja 6, exp. 6, f. 26.

74 Véase Rigoberto Álvarez Berrocal, "Las Manos de Gabriela Mistral. Poesía", en Repertorio Americano, vol. 7, núm. 15, Costa Rica, enero de 1924, p. 238. 
cuela Politécnica; y franquicia de derechos de examen y matrícula en sus Escuelas de Derecho y Ciencias Políiticas, Escuela de Medicina, de Farmacia, de Ingeniería y de Aviación. Cada uno de los demás Estados signatarios, agradeciendo la generosa oferta del Gobierno de Guatemala, se complacen en ofrecer, por su parte, a favor de los estudiantes de las Repúblicas hermanas, igual número de becas y las mismas facilidades en sus respectivos establecimientos oficiales de enseñanza. ${ }^{75}$

La experiencia mexicana de intercambio cultural parecía replicarse en otras latitudes.

\section{A MODO DE CONCLUSIÓN}

La última acción de la Secretaría de Educación Pública fue la repatriación de los becarios egresados a sus respectivos países. Este paso no sólo involucraba resolver nuevamente los temas logísticos, comprar pasajes, entregar viáticos, ${ }^{76}$ sino que hubo un especial énfasis en que la Secretaría de Relaciones Exteriores se hiciera partícipe de la inserción del nuevo profesionista en su tierra natal. Esto de alguna manera buscaba ser la última jugada dentro de los planes de propaganda del Estado posrevolucionario mexicano.

De todas maneras, en este ámbito las evaluaciones que se publicaron en $E l$ esfuerzo educativo... en 1928 eran lapidarias:

[... es de indicar la conveniencia de suprimir las pensiones en la forma que actualmente se conceden, para hacerlo de las dos únicas maneras que se justifican plenamente; por regla general, a los estudiantes pobres y aplicados, mediante dispensas en el pago de derechos escolares, y por excepción, como estímulo o como premio, a los que verdaderamente lo merecen por sus cualidades relevantes. ${ }^{77}$

75 Tratado de Confraternidad Centroamericana, 2 de julio de 1935, Art. X.

${ }^{76}$ A veces las cosas se complicaban. Por ejemplo, Rigoberto Álvarez Berrocal para volver a su localidad, debió ir de Veracruz a La Habana, de ese lugar a Colón en Panamá, de Colón a Puerto Limón en Costa Rica y desde ahí tomar un tren a su pueblo. Y para justificar el dinero solicitado a la SEP debió entregar cotizaciones de todos estos trayectos. Expediente de Rigoberto Álvarez Berrocal, AHSEP, Fondo Departamento Administrativo, caja 6, exp. 48.

77 Secretaría de Educación Pública, El esfuerzo..., p. 550. 
Los objetivos trazados en 1922 no fueron cumplidos y sólo durante los últimos 4 años se habían gastado 111280 pesos. En 1928 el Departamento de Intercambio Universitario, dependiente de la Universidad Nacional publicó un breve folleto con informaciones sobre la materia. ${ }^{78}$ Si algo se puede destacar de este texto, que retoma varios artículos del Boletín de la Unión Panamericana, es que no existe mención alguna a los pensionados centroamericanos. Como si fuera una realidad que las autoridades educativas quisieran olvidar.

Ahora bien, si revisamos el cuadro 1 podemos observar un dato que parece curioso; mientras todos los países van paulatinamente disminuyendo su cantidad de estudiantes en México, ya sea porque egresan, reprueban o desertan, solamente Nicaragua aumenta su número de becarios. Evidentemente la explicación no debemos buscarla en el ámbito académico, sino en la política exterior desarrollada por Plutarco Elías Calles, quien apoyó a los liberales nicaragüenses en su lucha contra la intervención norteamericana. De hecho, sus lazos con Augusto C. Sandino le generaron problemas con el gobierno de Estados Unidos.

De ese modo, antes de que se diera por concluido el proceso de intercambio académico iniciado en 1922, ya había comenzado a llegar a México una nueva olea de becarios. Desde ese momento, los cambios al sistema de pensiones sólo podrían hacerse sobre la marcha, con los nuevos estudiantes en las aulas universitarias.

78 Departamento de Intercambio Universitario, "Boletín Intercambio Universitario", Secretaría de Educación Pública, México, 1928. 
Cuadro 1 Estudiantes pensionados en México*

\begin{tabular}{|l|l|l|l|l|}
\hline Estudiantes & 1925 & 1926 & 1927 & 1928 \\
\hline Guatemala & 11 & 8 & 3 & 4 \\
\hline El Salvador & 8 & 7 & 5 & 4 \\
\hline Honduras & 11 & 8 & 5 & 5 \\
\hline Nicaragua & 6 & 8 & 10 & 13 \\
\hline Costa Rica & 6 & 4 & 3 & 2 \\
\hline Venezuela & 1 & 1 & 1 & $\ldots$ \\
\hline
\end{tabular}

* Con base en información de El esfuerzo educativo...

Cuadro 2 Resultados académicos de los estudiantes pensionados*

\begin{tabular}{|l|l|l|l|l|}
\hline Año & aprobaron & desertaron & terminaron & reprobaron \\
\hline 1925 & 34 & 7 & 1 & 1 \\
\hline 1926 & 25 & 3 & 8 & - \\
\hline 1927 & 34 & 7 & 1 & 3 \\
\hline
\end{tabular}

* Con base en información de El esfuerzo educativo...

Recibido: 30 de mayo, 2012. Aceptado: 6 de noviembre, 2012.

BibLIOGRAFÍA

Álvarez Berrocal, Rigoberto, "Las Manos de Gabriela Mistral. Poesía", en Repertorio Americano, vol. 7, núm. 15, Costa Rica, enero de 1924, p. 238.

Azuela de la Cueva, Alicia, Arte y poder. Renacimiento artístico y revolución social. México 1910-1945, México, El Colegio de Michoacán/FCE, 2005.

Bazant, Mílada, "Estudiantes mexicanos en el extranjero: el caso de los hermanos Urquidi", en Historia Mexicana, vol. 36, núm. 144, El Colegio de México, 1987, pp. 739-758. 
Buchenau, Jürgen, In the shadow of the Giant. The Making of Mexico's Central America Policy, 1876-1930, Alabama, The University of Alabama Press, 1996. Departamento De InTERCAMbio Universitario, "Intercambio de alumnos y de profesores”, en Boletín de la Secretaría de Educación Pública, t. I, núm. 2, México, $1^{\circ}$ de septiembre de 1922.

Departamento de InTERCAmbio Universitario, "Boletín Intercambio Universitario", México, SEP, 1928.

, "Los estudios universitarios en México y en el extranjero", en Revista de la Universidad de México, t. IV, núm. 21, 1932.

Díaz Vázquez, María del Carmen, "Intelectuales centroamericanos y el México posrevolucionario (1920-1930)", en Latinoamérica. Revista de Estudios Latinoamericanos, núm. 46, CIALC-UnAm, 2008, pp. 119-139.

, "Centroamérica en la estrategia diplomática mexicana: entre la política y la difusión cultural (1920 - 1932)", en Diálogos Revista Electrónica de Historia, número especial, 2008. En http://historia.fcs.ucr.ac.cr/dialogos.htm.

FABER, SeBASTIAN, Exile and Cultural Hegemony: Spanish Intellectuals in Mexico (1939-1975), Nashville, Vanderbilt University Press, 2002.

Fell, Claude, José Vasconcelos. Los años del águila, México, unam, 2009.

GARCIADIEGO, JAVIER, Rudos contra científicos. La Universidad Nacional durante la Revolución mexicana, México, El Colegio de México/unam, 1996.

Granados, Aimer, "La corriente cultural de la Jae en México: el Instituto Hispano Mexicano de Intercambio Universitario, 1925-1931", en Revista de Indias, vol. LXVII, núm. 239, Instituto de Historia (CSIC), 2007, pp. 103-124.

Herrera León, Fabián, "México y el Instituto Internacional de Cooperación Intelectual, 1926-1939", en Tzintzun. Revista de Estudios Históricos, núm. 49, Universidad Michoacana de San Nicolás de Hidalgo, 2009, pp. 169-200.

LIDA, ClaRA, "El perfil de una inmigración: 1821-1939", en Clara Lida [comp.], Una inmigración privilegiada. Comerciantes, empresarios y profesionales españoles en México en los siglos xIx y XX, Madrid, Alianza Editorial, 1994, pp. 25-51. 
López DE LA VeGa, Lucila, "Las celebraciones de 1825: del patriotismo a la educación. Las becas educativas de la Junta Cívica", en Historia Mexicana, vol. 60, núm. 237, El Colegio de México, 2010, pp. 641-655.

Loyo, Engracia, Gobiernos revolucionarios y educación popular en México, 1911-1928, México, El Colegio de México, 1999.

MACHUCA BeCERRA, ROBERTO, América Latina y el Primer Congreso Internacional de Estudiantes de 1921, México, 1996 (Tesis de licenciatura en Estudios Latinoamericanos, FFyL-unam), $184 \mathrm{pp}$.

MarSiske, Renate, Movimientos estudiantiles en la bistoria de América Latina, México, Centro de Estudios sobre la Universidad-unam/Plaza y Valdés, 1999. , "Crónica del movimiento estudiantil de México en 1929", en Revista Historia de la Educación Latinoamericana, núm.1, Universidad Pedagógica y Tecnológica de Colombia, 1998, pp. 35-62.

, "Los estudiantes en la Universidad Nacional de México: 1910-1928", en Varios autores, Los estudiantes. Trabajos de bistoria y sociología, México, CESu-unam, 1989.

McBeth, Brian S., "Foreign Support for Venezuelan Political Exiles during the Regime of Juan Vicente Gómez: the Case of Mexico, 1923-33", en The Historian, Phi Alpha Theta History Honor Society, 2007.

Melgar BaO, Ricardo, Redes e imaginario del exilio en México y América Latina, 1934-1940, Buenos Aires, Ediciones Libros en Red, 2003.

, "Redes del exilio aprista en México (1923-1924): una aproximación”, en Yankelevich, Pablo (coord.), México, país refugio. La experiencia de los exilios en el siglo XX, México, INAH/Plaza y Valdés, 2002.

Mistral, Gabriela, "Sobre el intercambio universitario", en El Mercurio de Santiago, 11 de mayo de 1924, p. 8.

"Resoluciones de la Primera Convención Internacional de Maestros", en Revista de Ciencias Económicas, año XVI, serie II, núms. 80-81, Buenos Aires, 1928, pp. 1805-1817.

Secretaría de Educación PúBlica, El esfuerzo educativo en México, la obra del gobierno federal en el ramo de educación pública durante la administración del presiente Plutarco Elías Calles (1924-1928). Memoria anali- 
tico-crítica de la organización actual de la Secretaría de Educación Pública, México, SEP, s/f.

, Memoria relativa al estado que guarda el ramo de educación pública, México, 1926, 1933, 1934.

, Noticia estadística sobre la Educación Pública en México, correspondiente al año de 1926, México, SEP, 1928.

Taracena Arriola, Arturo, "Latin Americans in Paris in the 1920s: the Anti-imperialist Struggle of the General Association of Latin American Students, 19251933", en Ingrid Fey y Karen Racine [eds.], Strange Pilgrimages. Exile, Travel and National Identity in Latin America, 1800-1990s, Wilmington, Jaguar Books, 2000, pp. 131-146.

The Guggenheim Foundation, "Announcement of Preliminary Gift of Three Million Dollars to Establish John Simon Guggenheim Memorial Foundation Endowing Fellowships for Advanced Study Abroad", en The High School Journal, vol. 8, núms. 3/5, University of North Carolina Press, marzo-mayo, 1925, pp. 48-50.

ThuRston, WaLter C., "Relations with Our Latin American Neighbors", en Annals of the American Academy of Political and Social Science, vol. 156, julio de 1931, pp. 116-125.

Universidad NaCional de México, "Intercambio universitario", 1928, en Suplemento del Boletín de la Universidad Nacional de México.

Wood, Bryce, "Scholarly Exchanges between Latin America and the United States", en Proceedings of the Academy of Political Science, vol. 30, núm. 4, Columbia University, 1972, pp. 123-140.

Yankelevich, PABlo, "En la retaguardia de la Revolución mexicana. Propaganda y propagandistas mexicanos en América Latina, 1914-1920" en Mexican Studies/Estudios Mexicanos, vol. 15, núm. 1, The University of California Press, invierno de 1999, pp. 35-71.

(coord.), México, país refugio. La experiencia de los exilios en el siglo XX, México, Plaza y Valdés/Conaculta, 1999.

Zuleta, María Cecilia, Los extremos de Hispanoamérica. Relaciones, conflictos y armonías entre México y el Cono Sur, 1821-1990, México, SRE, 2008. 